УДК 621.92

\title{
О ПРОЦЕССАХ ДРОБЛЕНИЯ В УДАРНО-ЦЕНТРОБЕЖНЫХ УСТАНОВКАХ
}

Абаржи И.И., докт. техн. наук

Институт технической теплофизики НАН Украины, ул Желябова, 2а, 03680, Украина

На основі теоретичного дослідження процесів дроблення в ударновідцентрових установках визначені як швидкості частинок в установці, так і зміна в часі та просторі густини розподілу числа частинок по poзмipax.

Библ. 14.
На основе теоретического исследования процессов дробления в ударно-центробежных установках определены как скорости частиц в установке, так и изменение во времени и пространстве плотности распределения числа частиц по размерам.
Based on the theoretical study of crushing processes in shockcentrifugal units are defined as the particle velocity in installation, and the change in time and spase the density of distribution of particle size.

Ключевые слова: дробление, ударно-центробежная установка, скорость частицы, плотность распределения числа частиц по размерам.

$A(l)$ - вероятность разрушения частицы в единицу времени;

$a$ - ускорение;

$a, b, c$ - константы;

$B(l / \gamma)$ - плотность распределения вероятности образования частицы дисперсной фазы размером $l$ при разрушении частицы, размером $\gamma$;

$C(\lambda)$ - постоянная интегрирования;

$C_{\phi}-$ фактор формы измельчаемых частиц;

$F(l)=F(x, l, t)=f(l) S$ - плотность распреде-ления числа частиц по размерам в сечении ударно-центробежного устройства;

${ }_{1} F_{1}(x ; y ; z)$ - вырожденная гипергеометрическая функция Гаусса;

$f_{20}(l)=F_{2}(l, 0)$, где $f_{20}(l)$ - начальное распределение функции $F_{2}(l, t)$;

$I(\alpha, \beta)$ - интеграл;

$i=1,2$;

$S$ - площадь сечения ударно-центробежного устройства;

$s$ - аргумент изображения Лапласа;

$T$ - температура;

$K_{1}$ и $K_{2}-$ коэффициенты в определении величин $A(l)$ и $B(l / \gamma)$;

$K_{r}$ - коэффициент распределения импульса между осколками при соударении частицы с рабочим органом;
$L$ - наибольший линейный размер частиц;

$P$ - давление;

$\mathrm{Re}$ - число Рейнольдса;

$f$ - плотность распределения числа частиц по размерам;

$f_{12}$ - сила взаимодействия между несущей и дисперсной фазами;

$l$ - линейный размер частиц;

$g$ - ускорение свободного падения;

$v_{1}, v_{2}$ - линейные значения скоростей несущей фазы и частицы размером $l$ соответственно вдоль оси $O X$;

$v_{2}^{\prime}(l / \gamma)$ - линейное значение скорости частицы размером $l$ твердой фазы, образовавшейся при разрушении частицы с линейным размером $\gamma$;

$\mathrm{v}_{2}{ }_{2}(1 / \gamma)-$ вектор скорости $v_{2}^{\prime}(l / \gamma)$;

$\mathrm{v}_{2}(\gamma)$ - вектор скорости частицы размером $\gamma$;

$\mathrm{V}_{\text {л }}$ - вектор скорости лопасти;

$x, y, z$ - аргументы функции ${ }_{1} F_{1}(x ; y ; z)$;

$t$ - время;

$t$ - переменная;

$z$ - переменная интегрирования;

$\gamma$-линейный размер частицы, $\gamma>l$;

$\alpha$ - объемное содержание фаз;

$\alpha, \beta, \mu$ - показатели степеней;

$\rho$ - плотность всей смеси; 
$\rho_{i}^{0}-$ истинная плотность $i-$ той фазы;

$\Phi(l, s)$ - изображение Лапласа $F(l, t)$;

$\varphi$ - скорость;

$\lambda$ - константа разделения;

$\chi$ - коэффициент температуропроводности;

$\tau$ - параметр;

\section{Индексы верхние:}

0 - истинная плотность составляющих смеси;

Основы теоретических исследований процессов дробления в различного рода установках заложены и существенно развиты еще во второй половине 20 столетия [1-7], однако особое значение имеют работы В.В. Кафарова и его сотрудников. Достаточно лишь указать на их монографии $[8,9]$ по системному анализу процессов химической технологии, в которых изложены не только подходы к этой проблеме, но и даны конкретные рекомендации по проверке и реализации идей, развитых ими в своих работах для различных типовых экспериментальных установок. Ниже, на этой основе, будет предпринята попытка описать в некотором приближении процессы дробления в установке, разработанной в ИТТФ НАН Украины и предназначенной (в зависимости от условий) как для дробления твердых веществ, так и их гранулирования. Она представляет собой горизонтально расположенный цилиндрический барабан, в котором имеются входные и выходные отверстия и в котором заключен вращающийся с определенной частотой ротор. На роторе прикреплены штифты, выполняющие как дробление, так и перемешивание твердых частиц. Их наличие $[8,9]$ позволяет отнести эту установку к ударно-центробежному типу и применить подходы, применяемые для описания протекающих в ней процессов.

Изложим основные посылки работ $[8,9]$, которых будем придерживаться и в данной статье. Рассматривается двухкомпонентная система, в состав которой входит как несущая (газ или жидкость), так и полидисперсная (подлежащая измельчению) твердая фазы. Несущая фаза [8,9] описывается моделью вязкой жидкости, т. е., с введением понятий тензоров вязких напряже-

\section{Индексы нижние:}

1 - несущая фаза;

2 - дисперсная фаза;

1, 2 - нумерация функций;

л - лопасть дробилки;

12 - взаимодействие между фазами 1 и 2;

$r$ - распределение импульса между осколками;

max - максимальное значение размера частицы;

' - скорость осколка частицы.

ний и тензоров поверхностных сил. Измельчение осуществляется только лишь за счет взаимодействия твердого материала с рабочими органами (размалыванием этой фазы за счет столкновения между отдельными частицами пренебрегается). Вводятся понятия объемного $\alpha_{i}$ содержания фаз, средних плотностей $\rho_{i}$, причем, очевидно, $\alpha_{1}+$ $\alpha_{2}=1, \rho_{1}+\rho_{2}, \rho_{i}=\rho_{i}^{0} \alpha_{i}$. Предполагается, что число частиц твердой фазы в системе достаточно велико и их распределение по размерам является непрерывным и характеризуется плотностью $f(1)$ распределения числа частиц по размерам $l$. Если их размеры находятся в пределах от $l$ до $l+d l$, то $f(l) d l \quad-$ число частиц линейных размеров в единице объема смеси. Тогда $\alpha_{i}=\int_{0}^{L} l f(l) d l, \rho=\int_{0}^{L} \rho_{i}^{0} l f(l) d l$.

Поскольку измельчение - процесс случайный, то вводятся понятия вероятности $A(l)$ разрушения частиц в единицу времени, а также плотности распределения вероятности $B(l / \gamma)$ образования частицы дисперсной фазы объемом при разрушении частицы, объемом $\gamma$. Тогда $B(l / \gamma) d l$ - вероятность образования частицы линейным размером от $l$ до $l+d l$ при разрушении частицы размером $\gamma$, так что $\int_{0}^{\gamma} B(l / \gamma) d l=1$. Вероятность столкновения частицы с рабочими органами полагается равной единице. Наконец, рассматриваются только парные столкновения.

Предполагается также, что имеет место локальное термодинамическое равновесие в пределах каждой из фаз. Это позволяет ввести для каждой из них свою температуру, удельную внутреннюю энергию, энтропию и другие термодинамические величины, и, следовательно, могут 
быть записаны соотношения Гиббса. Справедлива, наконец, также и гипотеза локальной однородности фаз, т.е., в любом локальном объеме вещество каждой фазы однородно вплоть до поверхности раздела, так что энергия и энтропия фазы пропорциональны массе, и эти величины являются аддитивными по массам фаз.

Ниже мы будем придерживаться полученной в [8] на основе этих посылок и законов сохранения массы, импульса и энергии системы уравнений, описывающей процессы дробления и справедливой для различных типов устройств. Но при этом ограничимся рассмотрением так называемой модели идеального вытеснения с переменной линейной скоростью потоков. Эта модель соответствует структуре поршневого движения потоков обеих фаз, при котором их перемешивание в направлении движения потоков отсутствует (скорости потоков фаз в направлении вытеснения зависят от пространственной координаты $x$ и времени), а в точках сечения, перпендикулярного направлению движения, средняя плотность несущей фазы $\rho_{1}$ и плотность $f(1)$ распределения числа частиц по размерам одинаковы. Тогда, пренебрегая еще массовыми силами и изменениями внутренней энергии обеих фаз и рабочих органов, имеем [8]:

$$
\left.\begin{array}{c}
\frac{\partial \rho_{1} S}{\partial t}+\frac{\partial \rho_{1} v_{1} S}{\partial x}=0 \\
\frac{\partial F(l)}{\partial t}+\frac{\partial F(l) v_{2}(l)}{\partial x}==-F(l) A(l)+\int_{l}^{l_{\max }} f(\gamma) A(\gamma) B(l / \gamma) d \gamma \\
\rho_{1} S \frac{\partial v_{1}}{d t}+\rho_{1} v_{1} S \frac{\partial v_{1}}{\partial x}=-\alpha_{1} S \frac{\partial P}{\partial x}-\int_{0}^{l_{\max }} \rho_{2}^{0} C_{\phi} l^{3} F(l) f_{(12)} d l-\rho_{1} g S+ \\
+\int_{0}^{l_{\max }} \rho_{2}^{0} C_{\phi} l^{3} F(l) A(l) v_{2}(l) d l-\int_{0}^{l_{\max }} \rho_{2}^{0} C_{\phi} l^{3} \int_{l}^{l_{\max }} F(\gamma) A(\gamma) B(l / \gamma) v_{2}(l / \gamma) d \gamma d l \\
F(l) \frac{\partial v_{2}(l)}{d t}+F(l) v_{2}(l) \frac{\partial v_{2}}{\partial x}=-\frac{F(l)}{\rho_{2}^{0}} \frac{\partial P}{\partial x}+F(l) f_{(12)}-F(l) g+ \\
+\int_{l}^{l_{\max }} F(\gamma) A(\gamma) B(l / \gamma)\left[v_{2}(l / \gamma)-v_{2}(l)\right] d \gamma
\end{array}\right\}
$$

Заметим, что вектор $v_{2}^{\prime}(l / \gamma)$ определяется [8] при этом как:

$\mathrm{v}_{2}^{\prime}(l / \gamma)=\mathrm{v}_{\pi}+K_{r} \gamma^{3} l^{-3}\left[\mathrm{v}_{\pi}-\mathrm{v}_{2}(\gamma)\right]$.

Выражения для величин $A(l)$ и $B(l / \gamma)$ для такого же типа устройств имеют [8] вид: $A(l)=K_{1} l^{\alpha}$ и $B(l / \gamma)=K_{2} \gamma^{\beta}(l / \gamma)^{\mu}$, где коэффициенты $K_{1}$ и $K_{2}$ зависят от технических параметров устройства, в частности, от числа $n$ оборотов измельчителя, его диаметра $d$, угла $\varphi$ атаки лопасти и модифи- цированного критерия $\operatorname{Re}=n d^{2} \rho_{1} / \mu_{1}$. Показатели степеней $\alpha, \beta, \mu$ определяются [8] из сравнения расчетных данных с экспериментом.

Дальнейшие упрощения связаны с пренебрежением ряда эффектов, учитываемых в (1). Если пренебречь взаимодействием фаз $\left(f_{12}=0\right)$, массовыми силами $(g=0)$, а также изменениями импульса обеих фаз за счет измельчения, положив $v_{2}=v_{2}$, то (1) можно переписать как

$$
\begin{gathered}
\frac{\partial \rho_{1}}{\partial t}+\frac{\partial \rho_{1} v_{1}}{\partial x}=0 \\
\frac{\partial F(l)}{\partial t}+\frac{\partial F(l) v_{2}(l)}{\partial x}=-F(l) A(l)+\int_{l}^{l_{\max }} f(\gamma) A(\gamma) B(l / \gamma) d \gamma \\
\rho_{1} \frac{\partial v_{1}}{d t}+\rho_{1} v_{1} \frac{\partial v_{1}}{\partial x}=-\alpha_{1} \frac{\partial P}{\partial x} \\
\frac{\partial v_{2}(l)}{d t}+v_{2}(l) \frac{\partial v_{2}}{\partial x}=-\frac{1}{\rho_{2}^{0}} \frac{\partial P}{\partial x}
\end{gathered}
$$


В [8] при описании процессов измельчения в барабанных мельницах система (2) еще более упрощена: предполагалось, что перепадом давления по длине установки можно пренебречь, и имеет место структура идеального перемешивания. Ниже мы откажемся от этого положения и будем описывать рассматриваемый процесс на основе (2). Из (2) видно, что в рамках данного предположения процессы переноса несущей и дисперсной фаз независимы друг от друга и могут быть рассмотрены каждый в отдельности. Остановимся на исследовании процессов, связанных с дисперсной фазой. Тогда (2) перепишется как

$$
\left.\begin{array}{c}
\frac{\partial F(l)}{\partial t}+\frac{\partial F(l) v_{2}(l)}{\partial x}=-F(l) A(l)+\int_{l}^{l_{\max }} f(\gamma) A(\gamma) B(l / \gamma) d \gamma \\
\frac{\partial v_{2}(l)}{d t}+v_{2}(l) \frac{\partial v_{2}}{\partial x}=-\frac{1}{\rho_{2}^{0}} \frac{\partial P}{\partial x}
\end{array}\right\}
$$

Начальные и граничные условия системы (3) будут сформулированы ниже.

Рассмотрим второе уравнение в (3), полагая, что перепад давления по длине аппарата - величина постоянная. Тогда, если $-\left(\rho_{2}^{0}\right)^{-1} \partial P / \partial x=a$, где $[a]=\mathrm{M} / \mathrm{c}^{2}$, то

$\frac{\partial v_{2}(l)}{\partial t}+v_{2}(l) \frac{\partial v_{2}(l)}{\partial x}=a$,

и система характеристических уравнений для (4) в соответствии с [10] имеет вид:

$t^{\prime}(\tau)=d t / d \tau=1, x^{\prime}(\tau)=d x / d \tau=v_{2}, v_{2}^{\prime}=d v_{2} / d \tau=\alpha$. Отсюда следует, что

$d x / d \tau=v_{2}$, и $d v_{2} / d \tau=a$.

Если в начальный момент времени имеет место некоторое распределение скорости по оси $x$, т.е., $\left.v_{2}(x, t)\right|_{t=0}=v_{2}(x, 0)=\varphi_{1}(x)$, то из (4) и (4') вытекает, чT०

$v_{2}(x, t)=\varphi_{1}\left(q_{1}(x, t)\right)+a t$,

$q_{1}(x, t)=x-v_{2} t+a t^{2} / 2$.

Подстановка (5) в (4) показывает, что (5) и есть решение (4). Если $\varphi_{1}\left(q_{1}(x, t)\right)$ - постоянная величина, например, $\varphi_{1}\left(q_{1}(x, t)\right)=b_{1}$, то $v_{2}(x, t)=b_{1}+$ $a t$, и подстановка его в (4) подтверждает, что оно является решением (5). Таким образом, в этом случае скорость частиц зависит только от времени. При линейной же зависимости от своего аргумента, т.е., если $\varphi_{1}\left(q_{1}(x, t)\right)=b_{1}+c_{1} \cdot q_{1}(x, y)=$ $=b_{1}+c_{1} \cdot\left(x-v_{2} t+a t^{2} / 2\right)$, где $\left[\mathrm{c}_{1}\right]=1 / \mathrm{c}$, находим, что $v_{2}(x, t)=\left[b_{1}+c_{1} \cdot\left(x+a t^{2} / 2\right)+a t\right]\left(1+c_{1} t\right)^{-1}$.

Подстановка (6) в (4) убеждает, что (6) - решение (4), но для определенности следует наложить условие, что $c_{1}>0$.

Если же скорость дисперсной фазы на входе аппарата со временем меняется по закону $\varphi_{2}(t)$, т.е., $\left.v_{2}(x, t)\right|_{x=0}=v_{2}(0, t)=\varphi_{2} t$, то из (4) и (4') следует, что

$v_{2}(x, t)=\sqrt{\varphi_{2}^{2}\left(q_{2}(x, t)\right)+2 a x}$,

$q_{2}(x, t)=t-a^{-1}\left[v_{2}(x, t)-\sqrt{v_{2}^{2}(x, t)-2 a x}\right]$.

И, как видно, $q_{2}(x, t) \rightarrow t-\mathrm{x} / v_{2}(\mathrm{x}, t)$ при $a \rightarrow 0$, т.е., $q_{2}(x, t)$ определена, и в этих условиях $\varphi_{2}(x, t) \rightarrow \varphi_{2}\left(\frac{v_{2}(x, t) t-x}{v_{2}(x, t)}\right)$. Наконец, подстановка (7) в (4) показывает, что (7) - решение (4).

По аналогии с предыдущим случаем выявим вид $v_{2}(x, t)$ при некоторых частных зависимостях $\varphi_{2}(t)$. Если $\varphi_{2}(t)-$ постоянная величина, т.е., $\varphi_{2}(t)=b_{2}$, то из (7) вытекает, что $v_{2}(x, t)=\sqrt{b_{2}^{2}+2 a x}$, и его подстановка в (4) убеждает в правильности данного решения.

При линейной зависимости $\varphi_{2}(t)$ от своего аргумента, т.е., если $\varphi_{2}(t)=b_{2}+c_{2} q_{2}(x, t)=b_{2}+\left(t-\left\lfloor v_{2}(x, t)-\sqrt{v_{2}^{2}(x, t)-2 a x} \mid a^{-1}\right) \cdot c_{2}\right.$,

то после подстановки этого выражения в (7) имеем, что

$$
\begin{aligned}
& v_{2}(x, t)=\left[-\left(b_{2}+c_{2} t\right) c_{2}+1\right. \\
& \left.+\left(a-c_{2}\right) \sqrt{\left(b_{2}+c_{2} t\right)^{2}+2 x\left(a-2 c_{2}\right)}\right]\left(a-2 c_{2}\right)^{-1} .
\end{aligned}
$$

Можно показать, что при $a \rightarrow 2 c_{2}$ из (7') следует $v_{2}(x, t)=b_{2}+c_{2} t+c_{2} x\left(b_{2}+c_{2} t\right)^{-1}$ и $v_{2}(x, t) \rightarrow b_{2}+c_{2} t$ при $x \rightarrow 0$, т.е., вид функции $v_{2}(x, t)-$ линейный. Наконец, при $a \rightarrow 0$ из (7') имеем

$v_{2}(x, t) \rightarrow 0,5\left[b_{2}+c_{2} t+\sqrt{b_{2}^{2}+c_{2}^{2} t^{2}-4 c_{2} x}\right]$.

Таким образом, уравнения (5) и (7) определяют поведение $v_{2}(x, t)$ в зависимости от типа условий, начального или граничного.

Обратимся теперь к первому уравнению в (3), т.е., к определению функции $F(l)$. Переобозначим ее как $F(l)=F(x, l, t)$ с тем, чтобы подчеркнуть ее зависимость от продольной переменной $x$, времени $t$, линейного размера частицы $l$, и перепишем первое уравнение в (3) как 


$$
\begin{aligned}
& \frac{\partial F(x, l, t)}{\partial t}+\frac{\partial F(x, l, t) v_{2}(x, l, t)}{\partial x}= \\
& =-F(x, l, t) A(l)+\int_{l}^{l_{\max }} F(x, \gamma, t) A(\gamma) B(l, \gamma) d \gamma,
\end{aligned}
$$

причем в (8) функция $v_{2}(x, t)$ определяется (в зависимости условий) уравнениями (5) или (7).

Литературные данные [1-7] свидетельствуют о поиске так называемого самосохраняющегося решения уравнения (8). Суть его состоит в том, чтобы найти такие преобразования искомой функции и переменных, которые позволили бы свести (8) к уравнению с некоторой, связанной с $F(x, l, t)$, функцией, зависящей только от одной, a не от трех переменных. Тогда семейство кривых, описываемое обычно тремя переменными, трансформировалось бы, как это отмечено в [4], в одну кривую. Примером может служить обычное одномерное уравнение теплопроводности, в котором искомая функция - температура среды - зависит от пространственной координаты $x$ и времени $t$. Если в нем положить, например, $y=x / 2 \sqrt{\chi t}$, где $\chi$ - коэффициент температуропроводности, то исходное уравнение перепишется как уравнение для температуры, зависящей только от $y$. Но уже и из постановки вопроса видно, что это решение явилось бы асимптотикой истинного распределе- ния температуры, область применимости которой следует определять.

При наличии же трех переменных необходимо провести преобразование и самой искомой функции. Такой способ представлен в [6,7], но он носит сложный характер. Ниже при решении (8) мы применим обычную [11] методику.

В соответствии с [8], как это уже подчеркнуто выше, для ударно - центробежных устройств функции $A(l)$ и $B(l / \gamma)$ зависят только от $l$ и $\gamma$. Это позволяет применить при определенных условиях метод разделения переменных при решении (8). Положим в (8) $F(x, l, t)=F_{1}(x) F_{2}(l, t)$. Если, например, $v_{2}(x, t)=v_{20}(x)=\sqrt{b_{2}^{2}+2 a x, \text { то }}$

$$
\begin{aligned}
& F_{1}(x) \frac{\partial F_{2}(l, t)}{\partial t}+F_{2}(l, t) \frac{\partial F_{1}(x) v_{20}(x)}{\partial x}= \\
& =-F_{1}(x) F_{2}(l, t) A(l)+F_{1}(x) \int_{l}^{l_{\max }} F_{2}(l, t) A(\gamma) B(l / \gamma) d \gamma .
\end{aligned}
$$

После деления обеих частей этого выражения на $F_{1}(x) F_{2}(l, t)$ приходим к

$$
\begin{aligned}
& \frac{1}{F_{1}(x)} \frac{\partial F_{1}(x) v_{20}(x)}{\partial x}=-\frac{1}{F_{2}(l, t)} \frac{\partial F_{2}(l, t)}{\partial t}-A(l)+ \\
& 1+\frac{1}{F_{2}(l)} \int_{l}^{l_{\max }} F_{2}(\gamma, t) A(\gamma) B(l / \gamma) d \gamma .
\end{aligned}
$$

Поэтому из (9) следует, что

$$
\left.\begin{array}{c}
\frac{1}{F_{1}(x)} \frac{\partial F_{1}(x) v_{20}(x)}{\partial x}=-\lambda^{2} \\
-\frac{1}{F_{2}(l, t)} \frac{\partial F_{2}(l, t)}{\partial t}-A(l)+\frac{1}{F_{2}(l)} \int_{l}^{l_{\max }} F_{2}(\gamma, t) A(\gamma) B(l / \gamma) d \gamma=-\lambda^{2}
\end{array}\right\} .
$$

Из первого уравнения в (10) имеем, что в этом приближении

$$
F_{1}(x, t)=C(\lambda)\left(b_{2}^{2}+2 a x\right)^{-1 / 2} \exp \left(-\lambda^{2} a^{-1}\left(b_{2}^{2}+2 a x\right)^{1 / 2}\right),
$$

где постоянная интегрирования $C(\lambda)$ зависит, очевидно, от $\lambda$.

Применим ко второму уравнению (10) преобразование Лапласа, полагая $\Phi(l, s)=\int_{0}^{\infty} F_{2}(l, t) \exp (-s t) d t$. Тогда после ряда преобразований получим, что

$$
\begin{gathered}
d \Phi(l, s) / d l+f(l, s) \Phi(l, s)=g(l, s), \\
f(l, s)=\left[-\frac{\mu}{l}+\frac{K_{1} K_{2} l^{\alpha+\beta}}{s-\lambda^{2}+A(l)}+\frac{1}{s-\lambda^{2}+A(l)} \frac{d}{d l}\left(A(l)-\lambda^{2}\right)\right], g(l, s)=\frac{1}{s-\lambda^{2}+A(l)}\left[\frac{d f_{20}}{d l}-\frac{\mu f_{20}(l)}{d l}\right] . \\
\Phi(l, s)=-\int_{l}^{l_{\max }} g(z, s) \exp \left(-\int_{z}^{l} f(z, s) d z\right) d z=-\int_{l}^{l_{\max }} g(z, s)\left(\frac{l}{z}\right)^{\mu} \frac{a_{1}+A(z)}{a_{1}+A(l)} \exp (-I(\alpha, \beta)) d z .
\end{gathered}
$$


Здесь $\alpha_{1}=s-\lambda_{2}, I(\alpha, \beta)=\int_{z}^{l} \frac{K_{1} K_{2} z^{\alpha+\beta} d z}{s-\lambda^{2}+A(z)}$. Интеграл $I(\alpha, \beta)$ выражается через гипергеометрическую функцию Гаусса, и, если $\beta=-1$, то
$I(\alpha,-1)=\int_{z}^{l} \frac{K_{1} K_{2} z^{\alpha-1}}{S-\lambda^{2}+A(z)} d z=\frac{K_{2}}{\alpha} \ln \frac{a_{1}+A(l)}{a_{1}+A(z)}$.

Поэтому (13) при $\beta=-1$ (с учетом выражения для $g(l, s))$ имеет вид:

$$
\Phi(l, s)=-\left(\frac{l}{l_{\max }}\right)^{\mu} f_{20}\left(l_{\max }\right) \frac{(s+b)^{\frac{K_{2}}{\alpha}}}{(s+c)^{\frac{K_{2}}{\alpha}+1}}+\frac{f_{20}(l)}{s+c}+K_{1} K_{2} l^{\mu} \int_{l}^{l_{\max }} \frac{f_{20}(z)\left(a_{1}+A(z)\right)^{\frac{K_{2}}{\alpha}-1}}{z^{\mu-\alpha+1}\left(a_{1}+A(l)\right)^{\frac{K_{2}}{\alpha}+1}} d z,
$$

где $b=K_{1}\left(l_{\max }\right)^{\alpha}+\lambda^{2}, c=K_{1} l^{\alpha}+\lambda^{2}$. Тогда из (14) следует, что

$$
\begin{aligned}
F_{2}(l, t) & =-f_{20}\left(l_{\max }\right) \exp \left(-\left(\lambda^{2}+K_{1} l_{\max }^{\alpha}\right) t\right)_{1} F_{1}\left[\frac{K_{2}}{\alpha}+1 ; 1 ; K_{1}\left(l_{\max }^{\alpha}-l^{\alpha}\right) t\right]+f_{20}(l) \exp \left(-\left(\lambda^{2}+K_{1} l^{\alpha}\right) t\right)+ \\
+ & \frac{\left.K_{1} K_{2} l^{\mu} t \exp \left(-\lambda^{2} t\right)\right)^{l_{\max }}}{\Gamma(2)} \int_{l} f_{20}(z) z^{-\mu+\alpha-1} \exp \left(-K_{1} z^{\alpha} t\right)_{1} F_{1}\left[\frac{K_{2}}{\alpha}+1 ; 2 ; K_{1}\left(z^{\alpha}-l^{\alpha}\right) t\right] d z
\end{aligned}
$$

Поскольку $F(x, l, t)=F_{1}(x) F_{2}(l, t)$, то

$$
\begin{gathered}
F^{*}(x, l, t)=C(\lambda)\left(b_{2}^{2}+2 a x\right)^{-1 / 2} \exp \left(-\lambda^{2} a^{-1}\left(b_{2}^{2}+2 a x\right)^{1 / 2}\right) \times \\
\times\left\{-f_{20}\left(l_{\max }\right) \exp \left(\left(-\lambda^{2}+K_{1} l_{\max }^{\alpha}\right) t\right)_{1} F_{1}\left[\frac{K_{2}}{\alpha}+1 ; 1 ; K_{1}\left(l_{\max }^{\alpha}-l^{\alpha}\right) t\right]+f_{20}(l) \exp \left(-\left(\lambda^{2}+K_{1} l^{\alpha}\right) t\right)+\right. \\
\left.+\frac{K_{1} K_{2} l^{\mu} t \exp \left(-\lambda^{2} t\right)^{l_{\max }}}{\Gamma(2)} \int_{l} f_{20}(z) z^{-\mu+\alpha-1} \exp \left(-K_{1} z^{\alpha} t\right)_{1} F_{1}\left[\frac{K_{2}}{\alpha}+1 ; 2 ; K_{1}\left(z^{\alpha}-l^{\alpha}\right) t\right] d z\right\} .
\end{gathered}
$$

Очевидно, (15) - частное решение (8), поэтому левая часть (15) обозначена через $F^{*}(x, l, t)$. Общее же решение - сумма таких частных решений по $\lambda$, т.е., $F(x, l, t)=\sum_{(\lambda)} F^{*}(x, l, t)$. Но так как $\lambda \in(-\infty, \infty)$, то это выражение следует ввести под интеграл [14], так что решение (8) имеет вид:

$$
F(x, l, t)=\int_{-\infty}^{\infty} F^{*}(x, l, t) d \lambda=\int_{-\infty}^{\infty} C(\lambda) \exp \left(-\lambda^{2} a^{-1}\left(b_{2}^{2}+2 a x\right)^{1 / 2}\right)\left(b_{2}^{2}+2 a x\right)^{-1 / 2} F_{2}(l, t) d \lambda .
$$

Для определения $C(\lambda)$ запишем значение функции $F(x, l, t)$ на входе в систему:

$$
F^{* *}(l, t)=\left.F(x, l, t)\right|_{x=0}=\int_{-\infty}^{\infty} F^{*}(0, l, t) d \lambda=\int_{-\infty}^{\infty} C(\lambda) \exp \left(-\lambda^{2} a^{-1} b\right) b^{-1} F_{2}(l, t) d \lambda .
$$

С другой стороны, $F^{* *}(l, t)$ можно разложить [14] в ряд Фурье:

$$
F^{* *}(l, t)=\frac{1}{2 \pi} \int_{-\infty}^{\infty} d \lambda \int_{-\infty}^{\infty} F^{* *}(\xi, t) \cos \left(\lambda^{2}(\xi-l) / v_{n}\right) d \xi .
$$

Из сравнения (17) и (18) и определяется $C(\lambda)$.

\section{Bыводы}

1. Определены скорости частиц при различных начальных и граничных условиях.

2. В приближении идеального вытеснения с переменными линейными скоростями потоков измельчаемых частиц и несущей фазы определен вид функции распределения частиц.

\section{ЛИТЕРАТУРА}

1. Гришаев И.Г., Классен П.В., Цетович А.Н. Особенности гранулирования минеральных удобрений методом окатывания.// Теоретические основы химической технологии. - 1977. - Т.11, №3. C. $437-443$.

2. Рахлин 3.Н., Гусев Ю.И., Мазур Г.Л. Закономер- 
ности роста гранул в барабанных грануляторах. // Теоретические основы химической технологии. 1975. - T.9, № 1. - С.129 - 131 .

3. Классен П.В., Гришаев И.Г. Основы техники гранулирования.-М.; Химия, 1982.-272 с.

4. Kapur $P$. The crushing and layering mechanism of granule growth. // Chem. Eng. Science. - 1971. V. 26, № 7. - P. 1093 - 1099.

5. Kapur $P$. Self - preserving size spectra of comminuted particles. // Chem. Eng. Science. 1973. - V. 27, № 3. - P. 425 - 431.

6. Ramabhadran T.E. and Seifeld J.H. Self preserving theory of particle systems. // Chem. Eng. Science. - 1975. - V. 30, № 7. - P. 1019 - 1025.

7. Pulvermacer B. and Ruckenstein E. Similarity solutions of population balance. // Journal of Colloid and Interface Science. - 1974. - V. 46, № 3. P. $1027-1033$.

8. Кафаров В.В., Дорохов И.Н., Арутюнов С.Ю. Системный анализ процессов химической технологии. Процессы измельчения и смешения сыпучих материалов. - М.; Наука, 1985. - 440 с.
9. Кафаров В.В., Дорохов И.Н., Кольичова Э.М. Системный анализ процессов химической технологии. Энтропийный и вариационный методы неравновесной термодинамики в задачах химической технологии. - М.; Наука, 1988. - 367 с.

10. Камке Э. Справочник по дифференциальным уравнениям в частных производных первого порядка. - М.; Наука, 1966. - 266 с.

11.БорщеевскийЮ.Т., ПроцышинБ.Н., АбаржиИ.И., Гордиенко П.В. Исследования процессов грануляции в грануляторах барабанного типа. //Промышленная теплотехника. - 2002. - Т.24. №2. C. $69-74$.

12. Бейтмен Г. и Эрдейи А. Высшие трансцендентные функции. Гипергеометрическая функция. Функции Лежандра. - М.; Наука, 1965. 296 c.

13. Градитейн И.С. и Рыжик И.М. Таблицы интегралов, сумм, рядов и произведений. - М.; Наука, 1971. - 1108 с.

14. Смирнов В.И. Курс высшей математики. Т.II. М.; Наука, 1967. - 656 с. 


\section{PROCESSES OF MATERIAL FRAGMENTATION IN SHOCK- CENTRIFUGAL DEVICES}

\section{Ivan I. Abarzhi}

Institute of Engineering Thermophysics of the National Academy of Sciences of Ukraine, vul. Zhelya-bova, 2a, Kyiv, 03680, Ukraine

We consider the processes of material fragmentation and crushing in shock - centrifugal facili-ties in approximation of an ideal replacement. In this approximation, the flow rate of the particle flux of fragmented material (e.g. dispersed phase) and the flow rate of the flux of material of the carrier phase are linear and depend on spatial coordinate and time. At the same time, the interactions of the carrier and dispersed phases of the material flow, the body forces and the change of momentum due to the fragmentation and grinding are neglected. This allows one consider the processes if transport of the carrier and dispersed phases of the flow as independent of one another. We further assume that the pressure change is a constant value at length scales comparable to the facility length scale. We derive analytical expressions for the particle velocities for various initial and boundary conditions. The equation is solved explicitly for distribution function in space and time by applying the method of separation of variables.

References 14.

Key words: crushing processes, shock-centrifugal units, particle velocity, the density of distribution of particle size.

1. GrishaevI.G., Klassen P.V., Tsetovich A.N. Features of granulation of mineral fertilizers the method of rolling . // Theoreticheskie osnovi himicheskoi tehnologii. - 1977. - V.11, №3. - P. 437 - 443.

2. Rahlin ZN., Gusev Yu.I., Mazur G.L. Conformities to law of growth of granules are in granulating drums. // Theoreticheskie osnovi himicheskoi tehnologii. - 1975. - V.9, №3. - P. 129 - 131 .

3. Klassen P.V., Grishaev I.G. Bases of technique of granulation. - M.; "Himiia", 1982.-272 p.

4. Kapur $P$. The crushing and layering mechanism of granule growth. // Chem. Eng. Science. - 1971. V. 26, № 7. - P. 1093 - 1099.

5. Kapur $P$. Self - preserving size spectra of comminuted particles. // Chem. Eng. Science. 1973. - V. 27, № 3. - P. 425 - 431.

6. Ramabhadran T.E. and Seifeld J.H. Self preserving theory of particle systems. // Chem. Eng. Science. - 1975. - V. 30, № 7. - P. 1019 - 1025.

7. Pulvermacer B. and Ruckenstein E. Similarity solutions of population balance. // Journal of Colloid and Interface Science. - 1974. - V. 46, № 3. P. $1027-1033$.

8. Kafarov V.V., Dorokhov I.N., Arutyunov S.Yu. System analysis of processes of chemical technology. Processes of growing and mixing of friable materials shallow. - M.; Nauka, 1985. - 440 p.

9. Kafarov V.V., Dorokov I.N., Kol'cova E.M. System analysis of processes of chemical technology. The entropy and variational methods of non-equilibrium thermodynamics in chemical tehnologii problems. M .; Nauka, 1988. - 367 p.

10. Kamke E. Reference book on differential equalizations in the partials of the first order. - M.; Nauka, 1966. - 266 p.

11. Barshchevski Yu.T., Procyshin B.N., Abarzhi I.I., Gordienko P.V. Researches of processes of granulation in granulyatorakh of drum type. // Promyshlennaya Teplotekhnika. - 2002. - V.24. № 2. - P. $69-74$.

12. Bateman G. and Erdelyi A. Higher transcendental functions. Hypergeometrical function. Functions of Legendre. - M.; Nauka, 1965. - 296 p.

13. Gradshtein I.S. and Ryzhik I.M. Tables of integrals, sums, series and products. - M.; Nauka, 1971. $-1108 \mathrm{p}$.

14. Smirnov V.I. Course of higher mathematics. V. II. - M.; Nauka, 1967. -656 p.

Получено 23.05.2016

Received 23.05.2016 